\title{
An Enhanced Obstetrics Track for a Family Practice Residency Program: Results from the First 6 Years
}

\author{
W. Suzanne Eidson-Ton, MD, MS, Jim Nuovo, MD, Blanca Solis, MD, \\ Kristine Ewing, MD, Heather Diaz, MD; and Lloyd H. Smith, MD, PhD
}

Background: Advanced training in obstetrics for family physicians occurs through a variety of methods. The program described has developed an obstetrics track for family practice residents.

Methods: Five residents have completed the 4-year residency program with enhanced obstetric training developed, and the results, in terms of procedural experience and examination scores, have been reviewed.

Results: These 5 family physicians performed a similar number of obstetric procedures compared with their Obstetrics and Gynecology resident counterparts, and they performed as well as their family medicine resident counterparts on national in-service examinations.

Conclusions: A 4-year enhanced obstetrics track is an effective means of improving the training of family medicine residents in obstetric procedures while maintaining the other fundamental training and residency review committee requirements for family medicine residents. (J Am Board Fam Pract 2005; 18:223-8.)

Approximately 22\% of active members of the American Academy of Family Physicians include obstetrics in their practices. Of those who provide obstetric services, a smaller number include such procedures as cesarean section $(4.1 \%)$, vacuum delivery $(19.2 \%)$, forceps delivery $(8.2 \%)$, and postpartum tubal ligation $(4.5 \%) .{ }^{1}$ Opportunities for residents to obtain obstetric skills include performing a fellowship (rural or maternal), entering a residency training program with a high obstetric volume, and/or using elective time for additional training. ${ }^{2,3}$ Eight years ago, our program developed an enhanced obstetrics (family practice/obstetrics or FP/OB) track. ${ }^{4}$ This was designed to allow residents to obtain a high volume of procedural and management skills in obstetrics but maintain their training in family practice. The curriculum is structured to ensure that residents meet all the family practice residency review committee (RRC) requirements.

Submitted, revised 7 February 2005.

From the Departments of Family and Community Medicine and Obstetrics and Gynecology, University of California, Davis, Sacramento, California. Address correspondence to W. Suzanne Eidson-Ton, MD, MS, Department of Family and Community Medicine, UC Davis, 4860 Y Street, Suite 2300, Sacramento, CA 95817 (e-mail: wetona.eidsonton@ucdmc.ucdavis.edu).
Key questions from the development of the enhanced obstetrics track included: What will be the procedural experience for $\mathrm{FP} / \mathrm{OB}$ residents? How will it compare with that of the obstetrics and gynecology (OB/Gyn) residents? How will FP/OB residents perform on the standardized tests in both specialties? Where will graduates of this program practice? We provide a review of our experience in the first 6 years of this program.

\section{Methods}

The setting of this program is the University of California, Davis Medical Center (UCDMC), which is a large regional referral hospital with a level 1 trauma center and a level 3 nursery. There are many referrals and transports to the obstetric service from the larger Northern California area, most of which is rural or semirural. The UCDMC labor and delivery currently provides services for more than 200 deliveries per month, and the cesarean section rate is close to $25 \%$, given the many preterm and high-risk births in addition to the many low-risk deliveries performed.

There is a traditional family practice residency program at the University of California, Davis, with approximately 42 residents at various levels. There is also an OB/Gyn residency with 4 to 5 residents per year during the period being re- 
ported. In the Family and Community Medicine (FCM) department, all 14 of the full-time faculty members participate in obstetric care, and 11 of them hold low-risk obstetric privileges in the labor and delivery area of the hospital. Two of the recent FP/OB graduates also hold cesarean section privileges, one with a primary appointment in the FCM department and one in the OB/Gyn department. Faculty and resident malpractice insurance is covered by the UCDMC malpractice coverage. There are documented and clear consultation guidelines between the FP and OB services. There is an OB/ Gyn attending physician in the Labor and Delivery area at all times. Monday through Friday, from the hours of 8 AM to 5 PM, this OB/Gyn faculty member is always a perinatologist.

The curriculum of the FP/OB program was well described in an article published in the $7 A B F P$ by Nuovo and Smith in 1999. ${ }^{4}$ In brief, FP/OB residents were assigned 5 month long training blocks on the obstetrics service in each of their 4 years of training (except for the initial resident, who had 4 months in postgraduate year (PGY) 1 and 5 months in each of the remaining 3 years). While on the obstetrics service, FP/OB residents participated in clinical services and curricular assignments identical to their OB/Gyn resident counterparts at each postgraduate year of training. For example, PGY-1 residents in both the OB/Gyn and FP/OB programs are expected to perform vaginal deliveries and postpartum tubal ligations, conduct rounds on all postpartum patients, triage patients newly admitted to labor and delivery, and cross-cover for all gynecology patients while on call. PGY-2 and PGY-3 residents perform cesarean sections, follow all the laboring and antepartum patients, and provide consultation to the emergency department. PGY-3 residents also spend more time in the outpatient $\mathrm{OB} / \mathrm{Gyn}$ clinic participating in specialty clinics (colposcopy, urogynecology, and vulvadynia) and general OB/Gyn care. PGY-4 residents function as chief residents in charge of the $\mathrm{OB}$ service, making plans for high-risk and antepartum patients, supervising the other residents, assisting in all surgical procedures on labor and delivery, and planning and presenting case discussions and morbidity and mortality discussions for didactic teaching. PGY-2 through PGY-4 residents also maintain a continuity clinic in the $\mathrm{OB} / \mathrm{Gyn}$ clinic, in addition to their family practice continuity clinic that is maintained all 4 years. Competition between
FP/OB and OB/Gyn residents was minimized by avoiding simultaneous assignments or by the use of shift assignments (day/night float shifts). Requirements for reading, conference attendance, and case presentation were identical for the FP/OB and $\mathrm{OB} /$ Gyn residents while on the obstetrics service. Differences occurred in the length of each training segment on the obstetrics service. OB/Gyn residents were assigned 10 - to 12 -week rotations, whereas FP/OB residents were assigned 4- to 10 week (usually 4- or 8-week) blocks. FP/OB residents were required to take the American Board of Family Medicine (ABFM) in-service examination each year, and both OB/Gyn and FP/OB residents were asked to take the Council on Resident Education in Obstetrics and Gynecology (CREOG) in-service written examination each year. Data regarding procedural experience were self-reported by each resident on a monthly basis according to the OB/Gyn categories established by the RRC. Continuity in family and community medicine by the FP/OB residents was maintained. The program was designed such that they would complete all their required rotations by the end of their 4 years of training, in addition to participating in continuity clinics and didactic training in keeping with their FP resident counterparts. The FP/OB residents did have somewhat less elective time available than the traditional FP residents, approximately 10 weeks versus approximately 16 weeks.

The funding for the extra year of training in the FP/OB program came from the UC Davis Health System. Before beginning the program, it was presented to the Graduate Medical Education Association Council, which approved the program. The Institutional Allocations Committee then approved the funding. Furthermore, before the start of the program, a specific proposal was submitted to the ABFM, the leadership of which also approved the program. At the time, there was no RRC approval process in place, given the innovative nature of the program. The RRC currently considers the program an integrated fellowship with extended training time.

\section{Results}

The current data include those from the 5 residents who graduated from the program in the years 2001, 2002, and 2003. There were no graduates from the FP/OB program in 2004. The data also include 
Table 1. Obstetrics Experience: Comparing FP/OB Residents with OB/Gyn Residents

\begin{tabular}{lccccccc}
\hline & $\begin{array}{c}\text { Spontaneous } \\
\text { Vaginal Delivery }\end{array}$ & $\begin{array}{c}\text { Forceps-Assisted } \\
\text { Vaginal Delivery }\end{array}$ & $\begin{array}{c}\text { Vacuum-Assisted } \\
\text { Vaginal Delivery }\end{array}$ & $\begin{array}{c}\text { Primary } \\
\text { Cesarean } \\
\text { Section }\end{array}$ & $\begin{array}{c}\text { Repeat } \\
\text { Cesarean } \\
\text { Section }\end{array}$ & $\begin{array}{c}\text { Multifetus } \\
\text { Delivery }\end{array}$ & $\begin{array}{c}\text { High Risk* } \\
\mathrm{FP}^{\dagger}\end{array}$ \\
$\mathrm{OB}^{\ddagger}$ & 312 & 11 & 19 & 131 & 62 & 13 & 213 \\
$\mathrm{OB}^{\S}$ & 308 & 23 & 20 & 167 & 88 & 24 & 218 \\
$P$ value & 354 & 22 & 20 & 170 & 88 & 25 & 229 \\
$P$ value $^{\mathbb{I}}$ & 0.93 & 0.07 & 0.81 & 0.08 & 0.08 & 0.27 & 0.89 \\
\hline
\end{tabular}

* Admissions to the obstetrics service of women with diabetes, hypertension, cardiac disease, preterm labor, and third-trimester vaginal bleeding.

†'The mean of the $5 \mathrm{FP} / \mathrm{OB}$ residents' data.

‡ The mean of the $13 \mathrm{OB} / \mathrm{Gyn}$ residents' data.

$\$$ The mean of the $9 \mathrm{OB} / \mathrm{Gyn}$ residents who completed all 4 years of training at this institution.

" Student $t$ test performed comparing FP/OB with all OB/Gyn resident data.

"I Student $t$ test performed comparing FP/OB with OB/Gyn resident data from those who completed all 4 years at this institution.

those from the $13 \mathrm{OB} / \mathrm{Gyn}$ residents who graduated in the same period ( 9 of whom completed all 4 years of their residency at this institution and 4 of whom matriculated to this program after their internship year). There was one FP/OB resident accepted for the program in the initial year, and 2 residents have been accepted in all subsequent years. There were $5 \mathrm{OB} / \mathrm{Gyn}$ residents in the first year of the FP/OB program and 4 in the subsequent years of the study period. During the 4-year curriculum, the FP/OB and $\mathrm{OB} / \mathrm{Gyn}$ resident groups experienced different total training duration on the obstetrics service as follows: OB/Gyn residents had 56 weeks on service and an average of 3708 hours of hospital on-call duty, whereas FP/OB residents had 80 weeks on service with an average of 3024 hours of hospital on-call duty. FP/OB and OB/Gyn resident procedural experience is compared in Table 1. The Student $t$ test was used to compare the 2 groups for each procedure. The mean numbers of spontaneous vaginal deliveries and vacuum-assisted vaginal deliveries were very similar. Although there were modest differences between the FP/OB residents and the OB/ Gyn residents in the number of forceps deliveries, cesarean sections (primary and repeat), and multifetus deliveries, these differences were not statistically significant $(P>.05)$ and are most likely explained by the different number of hours on call in each group, because the OB/Gyn residents had significantly more calls while on non-OB rotations. Furthermore, FP/OB and OB/Gyn residents managed similar numbers of high-risk obstetric patients requiring hospital admission. High-risk admissions that were tabulated included admissions to the obstetrics service of women with diabetes, hypertension, cardiac disease, preterm labor, and thirdtrimester vaginal bleeding. In addition to those conditions for which data were collected, all residents were involved in the care of women who were admitted with other high-risk conditions typically cared for in a tertiary care referral hospital including trauma in pregnancy, pregnancies complicated by fetal anomalies, fetal hydrops, and maternal lupus. FP/OB residents performed ultrasound examinations as a routine part of obstetric care, learning to perform biophysical profiles, amniotic fluid index assessment, fetal biometry, and umbilical cord Doppler ultrasound examinations in keeping with their OB/Gyn counterparts. Global performance evaluations by OB/Gyn faculty for the FP/OB residents reflected satisfactory progress throughout the training period. Finally, FP/OB residents participated as PGY-4 residents in the training of other OB/Gyn residents through direct supervision and required presentations at morbidity and mortality conference, grand rounds, and other educational conferences. There were initial concerns by some of the OB/Gyn faculty regarding this aspect of the program, but there were no problems during the actual training, and evaluations of the teaching quality of the FP/OB chief residents was uniformly satisfactory.

FP/OB residents' performance on the CREOG in-service examination is presented in Table 2. For the ABFM in-service examination, the mean composite score for fourth-year FP/OB residents was greater than the 60 th percentile. The mean com- 
Table 2. Performance Data of FP/OB Residents: Cognitive Test Results-CREOG In-Service Examination

\begin{tabular}{|c|c|c|c|c|c|c|}
\hline \multirow[b]{3}{*}{ Training Year } & \multicolumn{6}{|c|}{ Percentage Correct } \\
\hline & \multicolumn{3}{|c|}{ Obstetrics } & \multicolumn{3}{|c|}{ Ambulatory/Primary Preventive Care } \\
\hline & FP/OB & OB/Gyn & $P$ Value & $\mathrm{FP} / \mathrm{OB}$ & OB/Gyn & $P$ Value \\
\hline $\mathrm{R} 1$ & 59.2 & 59.2 & 1.0 & 68.0 & 64.0 & .75 \\
\hline R2 & 60.4 & 66.6 & .03 & 66.6 & 69.9 & .22 \\
\hline R3 & 66.5 & 71.2 & .14 & 73.0 & 72.8 & .96 \\
\hline $\mathrm{R} 4$ & 66.5 & 72.4 & .07 & 77.0 & 71.4 & .13 \\
\hline
\end{tabular}

posite score for the third-year traditional track family practice residents in the program was 55 th percentile. There were few significant differences between FP/OB residents and OB/Gyn residents on the CREOG in-service examination with respect to the obstetrics and ambulatory components; however, the OB/Gyn residents tended toward higher scores on the obstetrics component, whereas the FP/OB residents tended toward higher scores on the ambulatory/primary preventive care component. Faculty evaluations of all 5 of the FP/OB graduates compared favorably with $\mathrm{OB} / \mathrm{Gyn}$ residents with respect to clinical management, surgical skills, and knowledge base. At the time, faculty members were using a global evaluation form, but the departments are currently working toward more complex competency-based standards of evaluation.

In addition to the specialized training in obstetrics that the $\mathrm{FP} / \mathrm{OB}$ residents received, they also received more training in gynecology than traditional track FP residents. They were exposed to gynecologic issues by virtue of their participation as members of the OB/Gyn team, particularly during night call and on weekends. FP/OB residents performed an average of 36 (range, 22 to 52) postpartum bilateral tubal ligations and 41 (range, 15 to 83) dilation and curettage procedures during their 4 years of training (although data were not available from 2 of the FP/OB graduates regarding dilation and curettage procedures). In addition, $\mathrm{FP} / \mathrm{OB}$ residents also received extensive outpatient gynecologic training through the OB/Gyn continuity clinics and specialty clinics in which they participated throughout their training. They performed many more outpatient gynecologic procedures, such as endometrial biopsies, intrauterine device placements, and colposcopy than their traditionally trained family practice colleagues. There was also opportunity for training in therapeutic abortion for those FP/OB residents who desired this skill.

Regarding the current practice settings of the 5 FP/OB graduates, 4 are involved in the training of family practice residents in obstetrics; of these, 3 hold faculty positions in training programs. Of the 5 graduates, 3 are practicing in county clinic/hospital settings, serving primarily low-income, underserved populations; one of them practices in a rural setting and the other 2 in semiurban settings. The only graduate not involved in teaching practices in a rural underserved community. All the graduates perform vacuum-assisted deliveries and all have secured cesarean section privileges, except for one graduate who has not sought these privileges. Four of the graduates perform postpartum tubal ligations and dilation and curettage procedures. Four of the graduates have given formal feedback regarding their satisfaction with their training in response to a letter asking them to write a brief statement about how their FP/OB training has been important to them and how it is affecting their current practice. All 4 believed that their training was superb and prepared them well for their current practice environments. One graduate wrote, "I feel that the training I received in $\mathrm{OB}$ was excellent. I have had an incredible first year out in practice, but it would have been terrifying without my past training." Another wrote, "I think that I received excellent training in obstetrics and in nonoperative gynecology."

Finally, the effect of the FP/OB program on the traditional FP residents was positive as well. Their quantitative obstetric and gynecologic experience remained the same before and after the start of the FP/OB program. The quality of obstetrics and gynecologic training of the traditional residents increased throughout the years of this program, quantitatively demonstrated by an increase in their 
ABFM in-service examination scores. The mean composite score of the traditional track senior residents for the gynecology component increased from 490 in 1997 (when the first FP/OB resident began as an intern) to 550 in 2002, and the composite score for the obstetrics component increased from 520 to 590 in the same period. This increase in knowledge was probably secondary to having access to FP/OB colleagues in clinic and in labor and delivery of whom traditional track FP residents could easily ask questions and obtain informal consultations.

\section{Discussion}

There is a need for family physicians who practice obstetrics. This need is especially seen in rural areas. $^{5,6}$ Providing for adequate training in procedures such as cesarean sections, vacuum deliveries, forceps, and postpartum tubal ligations is difficult in a traditional FP program. The enhanced obstetrics track was designed to give family practice residents obstetric training similar to that received by $\mathrm{OB} / \mathrm{Gyn}$ residents. We have documented that, given the curricular design in this track, our residents achieve at least $80 \%$ of the OB/Gyn residents' obstetrics experience. Differences in case numbers between the FP/OB and OB/Gyn groups may have been related to the $\mathrm{OB} / \mathrm{Gyn}$ residents receiving more continuous obstetrics experience (eg, in-hospital on-call duty during their gynecology rotations), thus increasing the total hours of training on labor and delivery. There was a lower level of forceps experience by $\mathrm{FP} / \mathrm{OB}$ residents compared with $\mathrm{OB} / \mathrm{Gyn}$ residents and a relatively low number of forceps deliveries by all residents, reflecting a nationwide trend of decreasing forceps use. An unexpected side effect of the time FP/OB residents spent as members of the $\mathrm{OB} / \mathrm{Gyn}$ team was enhanced training in general gynecology and women's health compared with residents in the traditional family practice track. There is no significant difference in standard cognitive test scores for obstetric knowledge (CREOG in-service examination) between FP/OB residents and the OB/Gyn residents in the obstetrics category. Residents in the FP/OB track performed at least as well on the ABFM in-service examination as other FP residents as demonstrated by their composite scores in their last year of training.

The primary difficulty in the maintenance of this program has been the recruitment of qualified and committed residents and retention of those residents after the first 1 to 2 years of the program. We have found that the applicant pool is not as large as was originally expected. Furthermore, this is certainly a rigorous training program, given the enhanced expectations for specific performance skills, as well as being an intense environment, including many high-risk obstetric patients, the care of whom is likely to be experienced as more stressful. Although we received 10 to 15 applications per year for the $\mathrm{FP} / \mathrm{OB}$ program, the number of individuals willing to commit to the 4-year program was much lower; in some years, the 2 training positions were not filled. Changes to the curriculum have been necessary with the new RRC requirements for $\mathrm{FP}$ residents since the onset of the program. The increased number of required clinic patient encounters and the requirement that all required rotations be completed in the first 3 years of training have necessitated a shift in the amount of time spent on the obstetric service during the first 3 years. The residents now have 4 months of obstetric training in the first and third years, 5 in the second, and 6 in the third. This allows them to complete all their required FP rotations in the first 3 years and see the required numbers of clinic patients each year.

Despite the above challenges, we have shown that the enhanced obstetrics track is an effective means of improving the training of family practice residents in obstetric procedures, with the overall obstetric training comparable with that of OB/Gyn residents and with significant training in primary care gynecology. At the same time, FP/OB residents are able to meet their RRC requirements and demonstrate excellent performance on the ABFM in-service examination by the end of their training.

We cannot formally compare the training offered in this program to the experiences of family practice graduates who complete a maternal/rural fellowship, because there are no published data on the outcomes of these programs. We do believe that the FP/OB program offers several advantages for those family practice trainees interested in enhanced obstetrics training. The integrated FP/OB program trains residents in a developmentally appropriate manner with regard to skill development and level of responsibility. The residents acquire more skills and responsibility as they progress throughout the 4 years in a manner similar to that of their OB/Gyn colleagues. The integrated nature 
of the program allows $\mathrm{FP} / \mathrm{OB}$ residents to gain substantial obstetrics training without having to neglect other aspects of family medicine for extended periods of time. Furthermore, the FP/OB training gives residents 18 to 20 months of obstetrics training rather than the 12 months of a yearlong fellowship. Finally, by virtue of participating extensively in the $\mathrm{OB} / \mathrm{Gyn}$ clinic and as members of the hospital OB/Gyn team, FP/OB residents have increased opportunity to learn gynecologic skills important in the practice of family medicine. Formal comparison of the 2 methods of offering enhanced obstetrics training would be valuable for future consideration.

We thank Rahman Azari, $\mathrm{PhD}$, for help with the statistical tests in this study.

\section{References}

1. Facts about Family Practice. Kansas City (MO): American Academy of Family Physicians; 2003.

2. Delzell JE Jr, Ringdahl EN. The University of Missouri rural obstetric network: creating rural obstetric training sites for a university-based residency program. Fam Med 2003;35:243-5.

3. Helton M, Skinner B, Denniston C. A maternal and child health curriculum for family practice residents: results of an intervention at the University of North Carolina. Fam Med 2003;35:174-80.

4. Nuovo J, Smith LH. Enhanced obstetrics training for family practice residents: a unique collaborative program. J Am Board Fam Pract 1999;12:409-12.

5. Colwill JM, Cultice JM. The future supply of family physicians: implications for rural America. Health Affairs 2003;22:190-7.

6. Cohen D, Guirguis-Blake J, Jack B, et al. Family physicians make a substantial contribution to maternity care: the case of the state of Maine. Am Fam Physician 2003;68:405. 